\title{
ON THE GEOMETRIC CONTINUED FRACTIONS IN POSITIVE CHARACTERISTIC
}

\author{
S. DrISS ${ }^{1}$, H. KTHIRI ${ }^{2}$ \\ ${ }^{1}$ Faculty of science of Sfax, BP 1171, 3000 Sfax, Tunisia. E., \\ sanadriss@yahoo.fr - \\ hassenkthiri@gmail.com
}

\begin{abstract}
In this paper we study another form in the field of formal power series over a finite field. If the continued fraction of a formal power series in $\mathbb{F}_{q}\left(\left(X^{-1}\right)\right)$ begins with sufficiently large geometric blocks, then $f$ is transcendental.

Key words and Phrases: Continued fractions; Formal power series; transcendental numbers.
\end{abstract}

\begin{abstract}
Abstrak. Dalam paper ini dikaji form jenis lain di lapangan deret pangkat formal atas lapangan hingga. Jika continued fraction dari suatu deret pangkat formal adalah geometric block, maka $f$ transendental.

Kata kunci: Continued fractions; deret pangkat formal; bilangan transendental.
\end{abstract}

\section{INTRODUCTION}

The diophantine approximation issue introduced by Khintchine in [8] checks whether an irrational algebraic number $x$ of degree $>2$ has a continued fraction expansion with unbounded partial quotients. This work investigates a variety of explicit examples of transcendental continued fractions.

The first result of this type was suggested by to Liouville [9], who constructed real numbers whose partial quotients sequence grows very fast, too fast for the numbers to be algebraic. Subsequently, many authors used deeper transcendency criteria from Diophantine approximation to construct other transcendental continued fraction classes of interest. Maillet [10], was the first to explore transcendental continued fractions with unbounded partial quotients. More investigation was made by Baker [2, 3], Davison [5].

2000 Mathematics Subject Classification: 11R04, 11A55, 40A15.

Received: 13-06-2017, accepted: 10-04-2018. 
Unfortunately, in the case of power series fields, we do not have up to now similar theorems to these of Roth [6] and Schmidt [15]. Thus, it is clear that the methods used in $[1,2]$ do not enable one to study a formal power series whose continued fraction expansion satisfies a specific property.

In 1976, Baum and Sweet [4] proved that the unique solution in $\mathbb{F}_{2}\left(\left(X^{-1}\right)\right)$ of the cubic equation

$$
X \alpha^{3}+\alpha+X=0
$$

has a continued fraction expansion with partial quotients of bounded degree. They observed that no real algebraic number of degree $\geq 3$ has yet been shown to have bounded or unbounded partial quotients.

In 1986, Mills and Robbins [11] provided an example of algebraic formal series over $\mathbb{F}_{2}\left(\left(X^{-1}\right)\right)$ with an unbounded sequence of partial quotients.

In 2004, Mkaouar [13] gave a similar result to the Baker one [2] concerning the transcendency of formal series over a finite field.

In 2006, Hbaib et al. [7] obtained a result which allows the construction of a family of transcendental continued fractions over $\mathbb{F}_{q}\left(\left(X^{-1}\right)\right)$ from an algebraic formal series of degree more than 2 .

This work is intended to give a new transcendency form of formal power series over a finite field which depend only on the specific form appearing in the sequence of partial quotients. More precisely, if the continued fractions expansion begins with sufficiently large geometric block, then $f$ is transcendental.

The present paper is organized as follows: In section 2, we introduce the field of formal power series and the continued fraction expansion over this field and we review some basic properties. In section 3, we state the main continued fractions beginning with arbitrarily large palindromes and we give some lemmas that to be used to prove our results and we the main theorem is finally proved (see Theorem $3.4)$.

\section{Field of FORMAL POWER SERIES $\mathbb{F}_{q}\left(\left(X^{-1}\right)\right)$}

Let $\mathbb{F}_{q}$ be the finite field of $q$ elements, $\mathbb{F}_{q}[X]$ the ring of polynomials with coefficients in $\mathbb{F}_{q}$ and $\mathbb{F}_{q}(X)$ its field of rational functions. Let $\mathbb{F}_{q}\left(\left(X^{-1}\right)\right)$ be the field of formal power series

$$
\mathbb{F}_{q}\left(\left(X^{-1}\right)\right)=\left\{f=\sum_{n \geq n_{0}} b_{n} X^{-n} ; b_{n} \in \mathbb{F}_{q} ; n_{0} \in \mathbb{Z}\right\} .
$$

Define the absolute value

$$
|f|= \begin{cases}q^{\operatorname{deg} f} & \text { for } f \neq 0 \\ 0 & \text { for } f=0 .\end{cases}
$$


Thus, $\mid$. | is not an archimedean absolute value over $\mathbb{F}_{q}\left(\left(X^{-1}\right)\right)$, that is :

$$
\begin{array}{ll}
|f+g| \leq \max (|f|,|g|) & \text { and } \\
|f+g|=\max (|f|,|g|) & \text { if }|f| \neq|g| .
\end{array}
$$

By analogy with the real case, we have a continued fraction algorithm in $\mathbb{F}_{q}\left(\left(X^{-1}\right)\right)$. A formal power series $f=\sum_{n \geq n_{0}} b_{n} X^{-n}$ has a unique decomposition as $f=[f]+\{f\}$ with $[f] \in \mathbb{F}_{q}[X]$ and $|\{f\}|<1$. The polynomial $[f]$ is called the polynomial part of $f$ and $\{f\}$ is called the fractional part of $f$. We can write for any $f \in \mathbb{F}_{q}\left(\left(X^{-1}\right)\right)$

$$
f=a_{0}+\frac{1}{a_{1}+\frac{1}{\ddots \cdot+\frac{1}{a_{n}+\frac{1}{\ddots}}}}=\left[a_{0}, a_{1}, a_{2}, \ldots\right]
$$

where $a_{0}=[f]$ and $a_{i}=\left[f_{i}\right] \in \mathbb{F}_{q}[X]$ with $\operatorname{deg}\left(a_{i}\right) \geq 1$ for any $i \geq 1$ and $f_{i}=$ $\frac{1}{\left\{f_{i-1}\right\}}$.

The sequence $\left(a_{i}\right)_{i \geq 0}$ is called the sequence of partial quotients of $f$ and we denote by $f_{n}=\left[a_{n}, a_{n+1}, \ldots\right]$ the $n^{\text {th }}$ complete quotient of $f$.

Let $f$ be an algebraic formal power series of minimal polynomial $P(Y)=$ $A_{m} Y^{m}+A_{m-1} Y^{m-1}+\ldots+A_{0}$ where $A_{i} \in \mathbb{F}_{q}[X]$. Set $H(f)=\max _{0 \leq i \leq m}\left|A_{i}\right|$ and $\sigma(f)=A_{m}$.

Now, we define two sequences of polynomials $\left(P_{n}\right)_{n \geq 0}$ and $\left(Q_{n}\right)_{n \geq 0}$ as follows

and

$$
P_{0}=a_{0}, \quad Q_{0}=1, \quad P_{1}=a_{0} a_{1}+1, \quad Q_{1}=a_{1}
$$

$$
P_{n}=a_{n} P_{n-1}+P_{n-2}, \quad Q_{n}=a_{n} Q_{n-1}+Q_{n-2}, \text { for any } n \geq 2 .
$$

We easily check that

$$
P_{n} Q_{n-1}-P_{n-1} Q_{n}=(-1)^{n-1}, \text { for any } n \geq 1,
$$

and

$$
\frac{P_{n}}{Q_{n}}=\left[a_{0}, a_{1}, a_{2} \ldots ., a_{n}\right], \text { for any } n \geq 0
$$

$\frac{P_{n}}{Q_{n}}$ is called the $n^{\text {th }}$ convergent of $f$ and it satisfies the following :

$$
\lim _{n \rightarrow \infty} \frac{P_{n}}{Q_{n}}=f=\left[a_{0}, a_{1}, \ldots, a_{n}, \ldots\right] .
$$

With the non-archimedean absolute value, we find the following important equality

$$
\left|f-\frac{P_{n}}{Q_{n}}\right|=\left|\frac{P_{n+1}}{Q_{n+1}}-\frac{P_{n}}{Q_{n}}\right|=\left|Q_{n} Q_{n+1}\right|^{-1}=\left|a_{n+1}\right|^{-1}\left|Q_{n}\right|^{-2} .
$$


In 1949, Mahler proved in [10] a similar result with Liouville's inequality concerning rational approximations to algebraic real numbers:

Theorem 2.1. Let $f \in \mathbb{F}_{q}\left(\left(X^{-1}\right)\right)$ be an algebraic series over $\mathbb{F}_{q}(X)$ of degree $n>1$. Then, there exists a positive real number $C$, depending only on $f$, such that

$$
\left|f-\frac{P}{Q}\right| \geq \frac{C}{|Q|^{n}}
$$

for all $P, Q \in \mathbb{F}_{q}(X)$, with $Q \neq 0$.

In 1976, Baum and Sweet gave [4] these results:

Theorem 2.2. Let $f \in \mathbb{F}_{2}\left(\left(X^{-1}\right)\right)$. Then

a-: If $|Q f-P|=2^{-(\operatorname{deg}(Q)+d)}, \operatorname{gcd}(P, Q)=1, d>0$, then for some $n$,

$$
\left\{\begin{array}{l}
Q=Q_{n} \\
P=P_{n} \\
\operatorname{deg}\left(a_{n+1}\right)=d
\end{array}\right.
$$

b-: If $|Q f-P|=\frac{1}{|Q|}, \operatorname{gcd}(P, Q)=1$, then for some $n,\left\{\begin{array}{l}P=P_{n}+P_{n-1} \\ Q=Q_{n}+Q_{n-1}\end{array}\right.$.

Moreover, Baum and Sweet gave in [4] some examples of cubic formal series in $\mathbb{F}_{2}\left(\left(X^{-1}\right)\right)$ whose sequence of partial quotients is bounded.

\section{MAIN RESULTS}

Before giving the main result, we need to give the following definition .

Definition 3.1. We say that $U$ is a geometric block of order $s$ if there exists a polynomial $P_{i}[x] \in \mathbb{F}_{q}[x]$ where $K[X]=P_{1} \ldots P_{i}$, for all $i \geq 1$, such that

$$
U=K \cdot K^{q} \cdot K^{q^{2}} \ldots . K^{q^{s}}
$$

Lemma 3.2. [14] Let $f=\left[a_{0}, a_{1}, \ldots\right]$ and $g=\left[b_{0}, b_{1}, \ldots\right]$ be two formal series having the same $n+1$ terms of partial quotients. Then

$$
|f-g| \leq \frac{1}{\left|Q_{n}\right|^{2}}
$$

Lemma 3.3. [7] Let $f$ and $g$ be two formal series of degree $d$ and $m$ respectively. If $g$ reduced and $f \neq g$, then

$$
|f-g| \geq \frac{1}{H(f)^{m}|g|^{d-2}|\sigma(g)|^{\max (m-1, m(d-m+2)-1)}} .
$$

Theorem 3.4. We suppose that for $f=\left[a_{0}, a_{1}, \ldots, a_{n}, \ldots\right] \in \mathbb{F}_{q}\left(\left(X^{-1}\right)\right)$, there exists an increasing sequence $n_{i}$ such that $\left(a_{i}\right)_{i \geq 1}$ begins with a geometric block of order $n_{i}$ for all $i \geq 1$, then $f$ is transcendental. 
Proof.

Assume that $f$ is algebraic of degree $d>2$ and the continued fraction of $f=\left[H_{n}, H_{n}^{q}, H_{n}^{q^{2}}, \cdots, H_{n}^{q^{\lambda_{n}}}, \cdots\right]$ begins with a block $\lambda_{n}$ geometric.

Let $g_{n}$ denote the continued fraction

$$
\left[H_{n}, H_{n}^{q}, H_{n}^{q^{2}}, \ldots, H_{n}^{q^{\lambda_{n}}}, H_{n}^{q^{\lambda_{n}+1}}, \ldots\right] .
$$

With $H_{n}=K_{1}^{(n)} \cdots K_{s_{n}}^{(n)}$, then

$$
g_{n}=p_{1}^{(n)}+\frac{1}{p_{2}^{(n)}+\frac{1}{\cdots+\frac{1}{p_{s_{n}}^{(n)}+\frac{1}{g_{n}^{q}}}}} .
$$

where $\left(\frac{p_{1}^{(n)}}{q_{1}^{(n)}}\right), \cdots,\left(\frac{p_{s_{n}}^{(n)}}{q_{s_{n}}^{(n)}}\right)$ is the sequence of convergents $K_{1}^{n} \cdots K_{1}^{s_{n}}$. An easy calculation ensures that $g_{n}$ satisfies the following equation

$$
q_{s_{n}} g_{n}^{q+1}-p_{s_{n}} g_{n}^{q}+q_{s_{n}-1} g_{n}-p_{s_{n}-1}=0 .
$$

where $\left(\frac{p_{n}}{q_{n}}\right)_{n \geq 1}$ is the sequence of convergents of $g_{n}$. Hence $g_{n}$ is algebraic of degree $q+1$ such that

$$
H\left(g_{n}\right)=\left|p_{s_{n}}\right|=\prod_{i=1}^{s_{n}}\left|K_{i}^{(n)}\right| .
$$

And

$$
\sigma\left(g_{n}\right)=\left|q_{s_{n}}\right|=\prod_{i=2}^{s_{n}}\left|K_{i}^{(n)}\right| .
$$

Therefore, according to Lemma 3.3, we can check, for sufficiently large $n$ that:

$$
\left|f-g_{n}\right| \geq \frac{1}{H(f)^{q+1}\left|g_{n}\right|^{d-2}\left|\sigma\left(g_{n}\right)\right|^{d(q+1)-q^{2}}} .
$$

So, according to the equality (3) and (4), we obtain

$$
\left|f-g_{n}\right| \geq \frac{1}{H(f)^{q+1}\left|a_{0}\right|^{d-2}\left|\prod_{i=2}^{s_{n}} K_{i}^{(n)}\right| d(q+1)-q^{2}} .
$$

Furthermore, $f$ and $g_{n}$ have the same first $\lambda_{n}$ partial quotients; hence Lemma 3.2 implies that

$$
\begin{aligned}
& \left|f-g_{n}\right| \leq \frac{1}{\left|q_{s_{n}}\right|^{2\left(1+q+q^{2}+\cdots+q^{\lambda_{n}}\right)}} . \\
& \left|f-g_{n}\right| \leq \frac{1}{\left|\prod_{i=2}^{s_{n}} H_{i}^{(n)}\right|^{2\left(\frac{q^{\lambda_{n}+1}-1}{q-1}\right)}} .
\end{aligned}
$$


Combining (6) and (7), we get

$$
H(f)^{q+1}\left|a_{0}\right|^{d-2}\left|\prod_{i=2}^{s_{n}} K_{i}^{(n)}\right|^{d(q+1)-q^{2}} \geq\left|\prod_{i=2}^{s_{n}} H_{i}^{(n)}\right|^{2\left(\frac{q^{\lambda} n+1}{q-1}\right)} .
$$

Hence, we conclude that

$$
\limsup _{n \rightarrow+\infty} \frac{q^{\lambda_{n}+1}-1}{q-1}<\infty, \text { the desired contradiction. }
$$

Then $f$ is transcendental.

We close this paper with the following example.

Example 3.5. Let $f \in \mathbb{F}_{2}\left(\left(X^{-1}\right)\right)$ such that $f=\left[H_{n}, H_{n}^{2}, H_{n}^{4}, \cdots, H_{n}^{2^{\lambda n}}\right]$, where $H_{i}=\left[k_{1}^{(n)}, k_{2}^{(n)}, \cdots, k_{s_{n}}^{(n)}\right]$ such that $\lambda_{n}=n$, Then $f$ is transcendental.

\section{CONCLUDING REMARKS}

Recall that a polynomial $P \in \mathbb{F}_{q}[X][Y]$ is said to be reduced if $\operatorname{deg}\left(A_{m-1}\right)>$ $\operatorname{deg}\left(A_{i}\right)$ for any $i \neq m-1$, and an algebraic formal power series is reduced if its minimal polynomial reduced and $[f] \neq 0$.

- If $\left(\operatorname{deg}\left(a_{i}\right)\right)_{i \geq 0}$ is bounded, then $f$ is said to have a bounded continued fraction expansion.

- The expansion is finite if and only if $f \in \mathbb{F}_{q}(X)$.

- The sequence of partial quotients of $f$ is ultimately periodic if and only if $f$ is quadratic over $\mathbb{F}_{q}(X)$.

- Let $f$ be a quadratic power series over $\mathbb{F}_{q}(X)$. Then $f$ admits a purely periodic continued fraction expansion if and only if $f$ is reduced.

\section{REFERENCES}

[1] B. Adamczewski and Y. Bugea., On the Maillet-Baker continued fractions, J. Reine Angew. Math., 606 (2007), 105-121.

[2] A. Baker., Continued fractions of trascendental numbers, Mathematika., 9 (1962), 1-8.

[3] A. Baker., On Mahler's classification of transcendental numbers, Acta Math., 111 (1964), 97120.

[4] L.E. Baum and H.M. Sweet., Continued fractions of algebraic power series in characteristic 2,Ann. Math., 103 (1976), 593-610.

[5] J. L. Davison., A class of transcendental numbers with bounded partial quotients, In In $R$. A. Mollin, ed., Number Theory and Applications, pp. 365371, Kluwer Academic Publishers, 1989.

[6] H. Davenport., K.F. Roth., Rational approximations to algebraic numbers, Mathematika., 2 (1955) 160167.

[7] M. Hbaib, M. Mkaouar and K. Tounsi., Un critère de transcendance dans le corps des séries formelles $\mathbb{F}_{q}\left(\left(X^{-1}\right)\right)$, J. Number Theory., 116 (2006), 140-149. 
[8] A. Khintchine., Continued fractions, Gosudarstv. Izdat. Tech-Teor. Lit. Moscow-Leningrad, $2^{\text {nd }}$ edition, 1949, (In Russian).

[9] J. Liouville., Sur des classes très étendues de quantités dont la valeur n'est ni algébrique ni même réductibles à des rationnelles algébriques, J. Math. Pures Appl., 16 (1851), 133-142.

[10] E. Maillet., Introduction à la théorie des nombres transcendants et des propriétés arithmétiques des fonctions, Gauthier-Villars., Paris, 1906.

[11] W.H. Mills and D.P. Robbins., Continued fractions for certain algebraic power series, J. Number Theory., 23 (1986), 388-404.

[12] M. Mkaouar., Fractions continues et séries formelles algébriques réduites, Port. Math., 58 (2001).

[13] M. Mkaouar., Transcendance de certaines fractions continues dans le corps des séries formelles, J. Algebra., 281 (2004), 502-507.

[14] O. Perron., Die Lehre von den Kettenbrüchen, Teubner, Leipzig, 1929.

[15] W.M. Schmidt., Diophantine approximation, Lecture Notes in Mathematics, vol. 785. Springer, Berlin (1980) 